appear in the second parenthesis as a factor only if $\delta_{4}$ contains $g$, and in the third parenthesis only if $\delta_{6}$ contains $g$, and so on. But since $\delta_{n}=1, g$ must be a unit. If the quantities in parenthesis are relatively prime it is well known that we can find $x_{2}, x_{4}, \cdots, x_{n}$ so that (3) is satisfied.

If $n$ is odd the procedure is just the same with the exception that the single term in the last parenthesis is automatically prime to those in the preceding parentheses which have been determined as above, and as before we have only to determine $x_{2}, x_{4}, \cdots, x_{n}$.

The above theorem not only proves the existence of the $x$ 's but also enables us to calculate them.

The Catholic University of America

\title{
ON CONTINUED FRACTIONS WHICH REPRESENT MEROMORPHIC FUNCTIONS*
}

BY H. S. WALL

1. Introduction. In this paper I shall give sufficient conditions in order that a continued fraction of the form

$$
\frac{b_{1}}{1}+\frac{b_{2} z}{1}+\frac{b_{3} z}{1}+\cdots
$$

with arbitrary real or complex coefficients not zero shall represent a meromorphic function of $z$. Van Vleck $\nmid$ has shown that a sufficient condition is the following:

$$
\lim _{n=\infty} b_{n}=0 .
$$

Stieltjes $\ddagger$ proved that (2) is necessary as well as sufficient when the $b_{n}$ are real and positive. Van Vleck $\S$ proved that when the $b_{n}$ are real and $b_{2 n} b_{2 n+1}>0$, and the roots of the denominators, $D_{2 n+1}$, of the $(2 n+1)$ th convergents of (1) have distinct limits, not zero, for $n=\infty$, then the condition (2) is necessary and sufficient in order that (1) shall represent a meromorphic function of

* Presented to the Society, April 15, 1933.

$\dagger$ E. B. Van Vleck, Transactions of this Society, vol. 2 (1901), pp. 476-483. † Stieltjes, Oeuvres, vol. 2, pp. 560-566.

$\S$ E. B. Van Vleck: Transactions of this Society, vol. 4 (1903), pp. 309-310. 
z. He thought, and so stated (without attempting proof), that the condition imposed upon the roots of $D_{2 n+1}$ is always satisfied when (1) is meromorphic and $b_{2 n} b_{2 n+1}>0$. I have supplemented Van Vleck's result ( $\$ 3)$ by constructing an example of a continued fraction (1) with real $b_{n}$ such that $b_{2 n} b_{2 n+1}>0$ which represents a meromorphic function of $z$ and converges everywhere except at the poles of that function, but for which we have $\lim \sup \left|b_{n}\right|>0$.

2. Sufficient Conditions. We shall now develop conditions that are sufficient in order that (1) be a meromorphic function of $z$. If we multiply (1) by $z$, and make the substitution $b_{1}=1 / a_{1}$, $b_{n}=1 /\left(a_{n-1} a_{n}\right),(n=2,3,4, \cdots)$, that continued fraction becomes

$$
\frac{z}{a_{1}}+\frac{z}{a_{2}}+\frac{z}{a_{3}}+\cdots .
$$

Let it be supposed that the $a_{n}$ are arbitrary real or complex numbers different from zero, and that $c$ is a constant so chosen that $g_{n} \equiv c-\left(a_{2}+a_{4}+\cdots+a_{2 n}\right) \neq 0$ for $n=0,1,2, \cdots$, $\left(g_{0}=c\right)$.

Put $\quad h_{n}=1 / g_{n}, \quad a_{1}^{\prime}=h_{0}, \quad a_{2 n+1}^{\prime}=h_{n}-h_{n-1}, \quad a_{2 n}^{\prime}=a_{2 n-1} / h_{n-1}^{2}$, $(n=1,2,3, \cdots)$; and denote by $A_{n} / B_{n}, A_{n}^{\prime} / B_{n}^{\prime}$ the $n$th convergents of the continued fractions

$$
c-\frac{z}{a_{1}}+\frac{z}{a_{2}}+\frac{z}{a_{3}}+\cdots
$$

and

$$
\frac{1}{a_{1}^{\prime}}+\frac{z}{a_{2}^{\prime}}+\frac{z}{a_{3}^{\prime}}+\cdots,
$$

respectively. The following relations hold:

$$
\begin{array}{ll}
A_{2 n-1}=h_{n} A_{2 n}^{\prime}-A_{2 n+1}^{\prime}, & \\
B_{2 n-1}=h_{n} B_{2 n}^{\prime}-B_{2 n+1}^{\prime}, & \\
A_{2 n-2}=A_{2 n-1}^{\prime} / h_{n-1}, & (n=1,2,3, \cdots), \\
B_{2 n-2}=B_{2 n-1}^{\prime} / h_{n-1}, & \left(a_{0}=h_{-1}=1\right) .
\end{array}
$$


These may be verified directly for small values of $n$, and the induction then completed with the aid of the fundamental recursion formulas for continued fractions.

Set

$$
\begin{aligned}
t_{1} & =1 / a_{1}^{\prime}=1 / h_{0} ; \\
t_{2 n+1} & =1 /\left(a_{2 n}^{\prime} a_{2 n+1}^{\prime}\right)=h_{n-1} /\left(a_{2 n-1} a_{2 n} h_{n}\right), \\
t_{2 n} & =1 /\left(a_{2 n-1}^{\prime} a_{2 n}^{\prime}\right)=h_{n-1} /\left(a_{2 n-2} a_{2 n-1} h_{n-2}\right),
\end{aligned}
$$

$(n=1,2,3, \cdots)$. Then (5) takes the equivalent form

$$
\frac{t_{1}}{1}+\frac{t_{2} z}{1}+\frac{t_{3} z}{1}+\cdots .
$$

The numerators and denominators, $N_{n}^{\prime}, D_{n}^{\prime}$ of the $n$th convergent of (8) are connected with the $A_{n}^{\prime}, B_{n}^{\prime}$ by the relations

$$
N_{n}^{\prime}=A_{n}^{\prime} /\left(a_{1}^{\prime} a_{2}^{\prime} \cdots a_{n}^{\prime}\right), \quad D_{n}^{\prime}=B_{n}^{\prime} /\left(a_{1}^{\prime} a_{2}^{\prime} \cdots a_{n}^{\prime}\right) \text {. }
$$

We shall need the following lemma.

LemMa.* If $\lim t_{n}=0,(8)$ and the equivalent continued fraction (5) represent a meromorphic function $f(z)$, and converge uniformly over every bounded closed region containing no poles of $f(z)$. If the series $\sum t_{n}$ converges absolutely, then there exist two entire functions $u(z), v(z)$, without common zero, such that

$$
\lim _{n=\infty} N_{n}^{\prime}(z)=u(z), \quad \lim _{n=\infty} D_{n}^{\prime}(z)=v(z),
$$

uniformly over every bounded region.

We are now prepared to prove the following theorem.

THEOREM 1. The continued fraction (3) $\dagger$ in which the $a_{n}$ are real or complex numbers different from zero, and for which the series $\sum t_{n}$ (see (7)) converges absolutely, has the following properties.

(a) The sequence of even convergents represents a meromorphic function $F(z)$, and converges uniformly over every closed region $R$ containing no poles of $F(z)$.

(b) Set $k_{n}=1 /\left(a_{2 n} k_{n-1}\right), p_{n}=\left(k_{n}-1\right) / t_{2 n+1}$. Let $R^{\prime}$ be an ar-

* Perron, Die Lehre von den Kettenbrüchen, 1st ed., pp. 345-346.

$\dagger(3)$ is equivalent to (1) except for the factor $z$. 
bitrary closed bounded region containing none of the poles of $F(z)$, and containing no limit points of the sequence $\left\{p_{n}\right\}$, within or upon the boundary. Then the sequence of odd convergents converges uniformly over $R^{\prime}$ to $F(z)$.

(c) At an isolated limit point $p$ of $\left\{p_{n}\right\}$, not a pole of $F(z)$, the sequence of odd convergents converges to $F(p)$ if for every infinite sequence $p_{n_{1}}, p_{n_{2}}, p_{n_{3}}, \cdots$ converging to $p$, the sequence $q_{n_{m}}$ $=p_{n_{m}} t_{2 n_{m}} p /\left(p_{n_{m}}-p\right),(m=1,2,3, \cdots)$, does not have the limit point -1 .

(d) If $\left\{p_{n}\right\}$ has more than one limit point, and if the sequence of odd convergents converges for a value of $z$, it must converge to $F(z)$.

Proof of (a). By (9) and the last two equations (6) it will be seen that the $2 n$th convergent of (3) is identical with $c-\left(N_{2 n+1}^{\prime} / D_{2 n+1}^{\prime}\right)$. Hence, under the hypothesis of the theorem, it follows by the lemma that the sequence of even convergents of (3) converges uniformly over $R$ to the meromorphic limit $F(z) \equiv c-f(z)$.

Proof of (b). By (9) and the first two equations (6) the $(2 n-1)$ th convergent of $(3)$ is equal to

$$
c-\frac{A_{2 n-1}}{B_{2 n-1}}=c-\frac{k_{n} N_{2 n}^{\prime}-N_{2}^{\prime}{ }_{n+1}}{k_{n} D_{2 n}^{\prime}-D_{2}^{\prime}{ }_{n+1}} .
$$

This may be written in the form

$$
c-\frac{A_{2 n-1}}{B_{2 n-1}}=c-\frac{p_{n} N_{2 n}^{\prime}-z N_{2 n-1}^{\prime}}{p_{n} D_{2 n}^{\prime}-z D_{2 n-1}^{\prime}} .
$$

From (10) and the lemma it readily follows that the sequence of odd convergents of (3) converges uniformly over $R^{\prime}$ to $F(z)$.

Proof of (c). Equation (10) may be written in the form

$$
c-\frac{A_{2 n-1}}{B_{2 n-1}}=c-\frac{N_{2 n-1}^{\prime}+q_{n} N_{2 n-2}^{\prime}}{D_{2 n-1}^{\prime}+q_{n} D_{2 n-2}^{\prime}},
$$

where $q_{n}=p_{n} t_{2 n} p /\left(p_{n}-p\right)$ when $z=p$. Since $p$ is an isolated limit point of the sequence $\left\{p_{n}\right\}$, not a pole of $F(z)$, it follows from (10) as in the proof of (b) that any infinite sequence of odd convergents corresponding to values of $n$ for which $\left\{p_{n}\right\}$ has not the limit point $p$ must converge to $F(p)$ at $z=p$. Again, if 
$p_{n_{1}}, p_{n_{2}}, p_{n_{3}}, \cdots$ is a sub-sequence of $\left\{p_{n}\right\}$ converging to $p$, and, as supposed, the sequence $q_{n_{m}},(m=1,2,3, \cdots)$, does not have the limit point -1 , it readily follows from (11) and the lemma that the corresponding sequence (11) converges to $F(p)$ at $z=p$. We may then conclude that the sequence of odd convergents of (3) converges to $F(p)$ at $z=p$.

Proof of (d). Let the sequence of odd convergents converge for a certain value of $z$. Since, by hypothesis, $\left\{p_{n}\right\}$ has more than one limit point, it must have a limit point different from $z$, and there is an infinity of values of $n$ for which $\left|z-p_{n}\right| \geqq \delta>0$. The sequence of odd convergents corresponding to these values of $n$ must converge to $F(z)$, and hence the sequence itself converges to $F(z)$.

The argument used in the proof of (a) is valid under the more general hypothesis $\lim t_{n}=0$. Hence if $\lim t_{n}=0$, whenever the continued fraction converges it must be a meromorphic function. Now when the $b_{n}$ are real and $b_{2 n} b_{2 n+1}>0$, or, what is the same thing, the $a_{n}$ are real and $a_{2 n} \neq 0, a_{2 n+1}>0$, Hamburger* showed that the sequence of odd convergents always converges uniformly over an arbitrary bounded closed region $R$ exterior to the real axis; and if the limit, $F_{1}(z)$, is not identical with $F(z)$, then both $F(z)$ and $F_{1}(z)$ are meromorphic, non-rational functions of $1 / z$. Consequently if $\lim t_{n}=0$, we must have $F(z) \equiv F_{1}(z)$, for otherwise $F(z)$ would have an infinity of poles in the vicinity of the origin, which is impossible since it is a meromorphic function of $z$. We therefore have the following result.

ThEOREM 2. Let the $a_{n}$ of $(3) \dagger$ be real and $a_{2 n+1}>0, a_{2 n} \neq 0$. Then if $t_{n}$ is defined as in (7), and $\lim t_{n}=0$, the continued fraction represents a meromorphic function $F(z)$, with poles lying on the real axis only. The continued fraction converges uniformly over an arbitrary closed bounded region exterior to the real axis. The sequence of even convergents converges uniformly over an arbitrary closed bounded region containing none of the poles of $F(z)$ within or upon the boundary.

\footnotetext{
* Hans Hamburger, Mathematische Annalen, vol. 82, pp. 120-164 and 168-187.

$\dagger(3)$ is equivalent to (1) except for the factor $z$ in (3). The condition $a_{2 n+1}>0, a_{2 n} \neq 0$ is equivalent to the condition $b_{2 n} b_{2 n+1}>0$.
} 
3. Representation of a Meromorphic Function. We proceed to prove that a continued fraction (1) with real $b_{n}$ such that $b_{2 n} b_{2 n+1}>0$, may represent a meromorphic function of $z$ when $b_{1}, b_{2}, b_{3}, \cdots$ does not converge to 0 . To construct such a continued fraction, let $r, s$ be real and positive and $r<1, r s>1$, $r^{3} s \leqq 1$, and take $b_{1}=1$, and

where

$$
\begin{aligned}
b_{4 n} & =(1 / r)\left(1 /\left(r^{3} s\right)\right)^{n / 2}, & & b_{4 n+1}=r(r / s)^{n / 2}, \\
b_{4 n+2} & =\sigma_{n}\left(1 /\left(s^{3} r\right)\right)^{n / 2}, & & b_{4 n+3}=\delta_{n}\left(1 /\left(r^{3} s\right)\right)^{n / 2},
\end{aligned}
$$

$\sigma_{n}=\left(1-r^{n+1}\right) s^{n+2} /\left[\left(1-s^{n+2}\right) s^{2}\right], \delta_{n}=\left(1-r^{n+2}\right) s^{n+2} /\left[\left(1-s^{n+2}\right) r^{2} t\right]$, $t=(r s)^{1 / 2},(n=0,1,2, \cdots)$. The continued fraction (1) with the above values of the $b_{n}$ is of the kind considered by Van Vleck. For $b_{2 n} b_{2 n+1}>0,(n=1,2,3, \cdots)$, and $b_{1}=1$, it will be seen that

$$
\lim \sup \left|b_{n}\right|=\left\{\begin{array}{l}
1 / r \text { if } r^{3} s=1 \\
\infty \text { if } r^{3} s<1
\end{array}\right.
$$

If we substitute $k z$ for $z, b_{n}^{\prime}$ for $k b_{n}, k>0$, we will obtain a continued fraction of the same kind but for which lim sup $\left|b_{n}{ }^{\prime}\right|$ can be made any positive number by choosing $k$ properly.

To prove that the continued fraction represents a meromorphic function, we shall apply Theorem 1 . Put $b_{1}=1 / a_{1}$, $b_{n}=1 /\left(a_{n-1} a_{n}\right),(n=2,3,4, \cdots)$, so that

$$
a_{2 n}=\left(b_{1} b_{3} b_{5} \cdots b_{2 n-1}\right) /\left(b_{2} b_{4} b_{6} \cdots b_{2 n}\right), \quad(n=1,2,3, \cdots) .
$$

I find that if $c=1 /(1-r)$,

$$
\begin{aligned}
a_{4 n+2} & =c t^{n^{2}+3 n}\left(1-s^{n+2}\right), \quad(n=0,1,2, \cdots), \\
a_{4 n} & =c t^{n^{2}+3 n}\left(1-r^{n+1}\right) / r^{n+1}, \quad(n=1,2,3, \cdots), \\
h_{2 n} & =1 /\left(c t^{n^{2}+3 n}\right), h_{2 n-1}=r^{n+1} /\left(c t^{n^{2}+3 n}\right), \\
k_{2 n} & =1 /\left(1-r^{n+1}\right), k_{2 n-1}=1 /\left(1-s^{n+1}\right), \\
p_{2 n} & =r^{n+1} t^{n} /\left(1-r^{n+1}\right), p_{2 n-1}=s^{n-1} t^{n-1} /\left[\sigma_{n-1}\left(1-s^{n+1}\right)\right], \\
t_{4 n+1} & =1 / t^{n}=t_{n+2}, t_{4 n+3}=\sigma_{n} s^{2} / t^{n}, t_{4 n+4}=\delta_{n} /\left(s^{2} t^{3 n}\right), \\
q_{2 n} & =\delta_{n-1} r^{2} t z /\left\{\left[r\left(r^{3} s\right)^{n / 2}+\left(r^{n+1}-1\right) z\right] s^{n+1}\right\} .
\end{aligned}
$$

Since $\lim t_{n}=0$, Theorem 2 may be applied to prove that the continued fraction (3) represents a meromorphic function $F(z)$ and converges uniformly over every closed bounded region exterior to the real axis. This theorem gives us no information re- 
garding the convergence of the sequence of odd convergents for points on the real axis. We therefore turn to Theorem 1, which is applicable here inasmuch as $\sum t_{n}$ converges absolutely.

When $r^{3} s<1$, the sequence $\left\{p_{2 n}\right\}$ converges to 0 , while $\left\{p_{2 n+1}\right\}$ increases numerically to $\infty$. Hence by (a) and (b) of Theorem 1, the continued fraction (3) converges uniformly over an arbitrary bounded closed region $R$ not containing the origin or a pole of $F(z)$. Since $q_{2 n}=p_{2 n} t_{4 n} p /\left(p_{2 n}-p\right)=0$ when $p=0$, so that $\left\{q_{2 n}\right\}$ does not have the limit point -1 , and since 0 is an isolated limit point of $\left\{p_{n}\right\}$, (c) of Theorem 1 is applicable at $z=0$.

When $r^{3} s=1, \lim p_{2 n}=r, \lim \left|p_{2 n+1}\right|=\infty$; and when $p=r$, $\lim q_{2 n}=0$. Hence in this case also the continued fraction (3) converges except at the poles of $F(z)$.

Now (1) and (3) are equivalent except for the factor $z$ in (3). It follows that if $z \neq 0$, (1) represents the meromorphic function $H(z)=F(z) / z$, and converges except at the poles (not $z=0$ ) of $H(z)$. It will be seen that $z=0$ is not a pole of $H(z)$, but that $H(0)=b_{1}=1 / a_{1}$. Since (1) converges to this limit when $z=0$, it follows that the point $z=0$ is not an exceptional point.

When $r^{3} s<1$, lim sup $\left|b_{n}\right|=\infty$, and, by a theorem of Van Vleck, ${ }^{*}$ the distance between the origin and the nearest root of $D_{n}$ (the denominator of the $n$th convergent of (1)) has no positive lower limit. It is interesting to exhibit this fact directly for the continued fraction under consideration. By (9) and (6), it will be seen that the roots of $D_{4 n-1}$ are the roots of

$$
P_{2 n} D_{4 n}^{\prime}-z D_{4 n-1}^{\prime} \text {. }
$$

Now this converges uniformly over a region containing the origin, and, since $\lim p_{2 n}=0$, its limit is $-z v(z)$, a function which has a zero at the origin. We conclude that an arbitrarily small vicinity of the origin must contain a zero of (12), and hence of $D_{4 n-1}$, provided $n$ is sufficiently large.

When $r^{3} s=1, \lim \sup \left|b_{n}\right|=1 / r$, and, by this same theorem of Van Vleck, the roots of $D_{n}$ cannot come within some minimum distance $d>0$ of the origin.

NORTHWESTERN UNIVERSITY

* Transactions of this Society, vol. 4 (1903), p. 209. 